\title{
Comparative Study on Subjective Evaluation of Color-to-Grey Conversion Method
}

\author{
Dwilya Makiwan $^{1}$ and Mario Koeppen ${ }^{2}$
}

\begin{abstract}
Humans use color not only to interpret or distinguish objects but also to identifying interest regions for analysis. One of the most well known methods for colorization is Pseudo-coloring; this is a method designed to assign arbitrary colors to grey levels by a fixed mapping. For applying a Pseudo-coloring technique that starts from a color image, a method for converting initial colors to grey tones is needed for assisting the conversion process and the conversion need to represent the appearances of the real color from digital images when the conversion finishes. There are many conversions these days but some of those conversions could not present the real color information from images. To avoid this, before beginning Pseudo-coloring, we must pinpoint which method is best used for grey transformation. The comparison result then will lead us to implement it to pseudo-color techniques. Here, we report about the results of comparative experiments on color to grey transformations, favoring the YPQ color space.
\end{abstract}

Keywords-Color Conversions, color mapping, pseudo-color, voting.

\section{INTRODUCTION}

$I^{2}$ $\mathrm{N}$ our life, vision and actions are influenced by geometry and color information. The assessment of color information also plays important role in object identification [2]. For example, we usually search for a known book not only based on the title but also we try to remember the color of the cover and then try to match it with our memory. This leads color into one of the most helpful tools for identification [2]. In this research, we will discuss the application of several schemes that can give enhancement effects and approach better design.

The use of color for human interpretation not only to improve the number of objects that can be distinguished but also color adds vivacity to images decreases boredom and improves the attention. Pseudo-coloring of gray scale images is a technique used to improve the detection of weak features, structures, and patterns in an image by providing image details that otherwise would not be noticed [1]. Before getting into pseudo-coloring, we need to find out which is the best method to use for grey transformation.

We believe that the best way to decide whether a method is good enough or not is based on what human visual can perceive well. Therefore, in this paper we present a study on comparing four famous methods of color to grey transformation.

Dwilya Makiwan ${ }^{1}$ Kyushu Institute of Technology, Department of Creative Informatics, Fukuoka, Japan.

Mario Koeppen ${ }^{2}$ Kyushu Institute of Technology, Department of Creative Informatics, Fukuoka, Japan.
This article is organized as follows: Section II will provide some background of color palette and color lookup table. Section III presents the comparison and evaluation of color to grey transformation and will briefly present the comparison result. On Section IV will give an extension topic of how a pseudo-color technique works. At the end, there will be short conclusion and discussion of future design of color to grey transformation_and pseudo-color.

\section{Color PALETTE AND Lut (COLOR LOOKUP TABLE)}

\section{A. Color Palette}

The RGB color space is the most applied computer representation of color image because all visible color can be represented by linear combination of three vectors (stands for each RGB value) [2].

RGB color space turned into an arrangement of a limited system colors that can be chosen and expressed as a palette. In the image processing system, the cost of high memory become a problem because full color display will make many applications cannot work very well. This happen because common color resolution for high quality images is 24 bits for each Red, Green, Blue channels, or $256=16777216$ colors [4]. We can solve the problem by using 8bit image and display at most 256 distinct colors at a time. Then the best thing to do is to make few color combination, called palette and distribute (map) the rest of the colors.

Palette as a solution can be used to display with reasonable accuracy any kind of image. This is done by selecting particular colors that contains RGB color space in small way, which means limiting the levels that the red, green, and blue have. Assigning more levels for the green components can do the limiting arrangement and less to blue components, this because normal human eyes has sensibility to RGB color, more sensitive to green compare to blue.

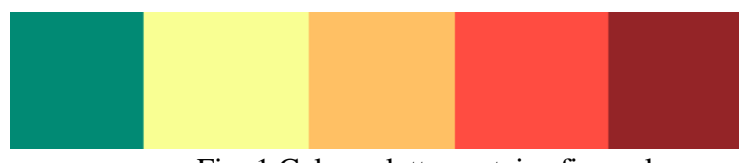

Fig. 1 Color palette contains five colors.

\section{A. Color Lookup Table}

As explained before, for full color display of RGB will have a memory problem but we can generate it by using eight bits of each RGB color (total 24 bits). Color LookUp Table contains this calculation. When a display needs to process a standard calculation, we can improve the performance by having it look up and LUT value instead of calculate it one by one. 


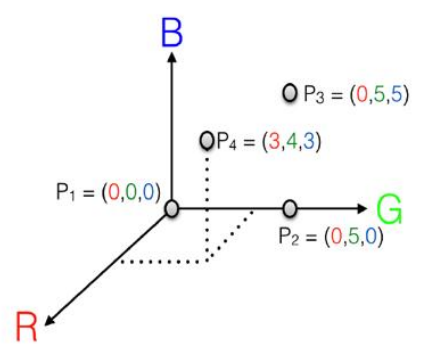

Fig. 2 RGB vectors for LUT

The time save calculation can be well explained below;

1) From the picture above, we have three axes belongs to Red, Green and Blue. Lets say the intersect point is the center where the values are $(0,0,0)$ for each $\mathrm{R}, \mathrm{G}, \mathrm{B}$ and call it $\mathrm{P}_{1}$.

2) Imagine the point moves along $G$ line without stepping into $\mathrm{R}$ and $\mathrm{B}$ territory. After taking five steps, the point will arrive in $\mathrm{P}_{2}$ means the R,G,B value will be $0,5,0$.

3) From $\mathrm{P}_{2}$ position, then move the point five steps upwards then we will get five for each color blue and green value. Here, the value changes in two-dimensional way and this changes two values at the same time.

4) Now move the $P_{3}$ to R territory and this move will get three dimension values that include red. Moving the value in three-dimensional is the way where the time safe calculation stands for. This because the point moves once but all three values will change.

Shortly, Color LookUp Table is a way to transform a range of input colors into another range of colors by simply present it as a table of cross-link index numbers of RGB that commonly used to determine the colors and intensity of a particular image. A common example would be a palette of 256 colors that is the addressed by 8 -bit pixel value.

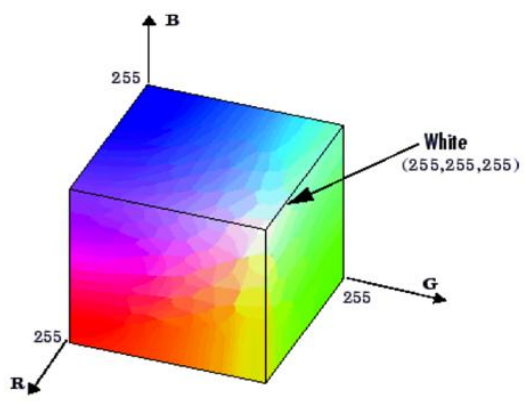

Fig. 3 RGB color LUT cube.

Since LUT provide color reproduction from RGB, the user's manipulation and color adjustments will come with some parameters such as brightness, chroma, and hue, which is important in order to get into the pseudo-coloring technique.

\section{COLOR TO GREY TRANSFORMATION}

In this section, we will first introduce what digital image and pixel is, and then explains about color to grey transformation process. We will also talk about a small experiment of comparing color to grey transformation methods and last is to explain about the comparison for each method.

A digital image is made up of picture elements called pixel. Most of digital image contains 24 bits of RGB system to color each pixel. Which means each pixel contains 256 color graduation of red, green and blue. For example the color cyan is 0 Red, 255 Green and 255 Blue. The dimensions of a digital photo are expressed in terms of pixel array (matrix of $M$ columns $\mathrm{x} N$ rows) and defined as coordinate $x$ and $y$. The coordinate system of image matrices defines $x$ as increasing from left to right and $y$ as increasing from top to bottom [3]. For example an image contains 800x600 pixels or 1520x1280 pixels where the first number counts the pixel from $x$ (width) of the image and the second number counts the pixel from $y$ (height) of the image.

Beside of RGB value, each pixel also contains another important parameters, intensity, are needed to truly define an image [5]. The image will have various shades of grey when the intensity is the same. The intensity range goes from black to white. While in color image, the intensity will have various ranges of colors from darkest to lightest of red, green and blue. When these color intensities combine each other, then it will produce color image.

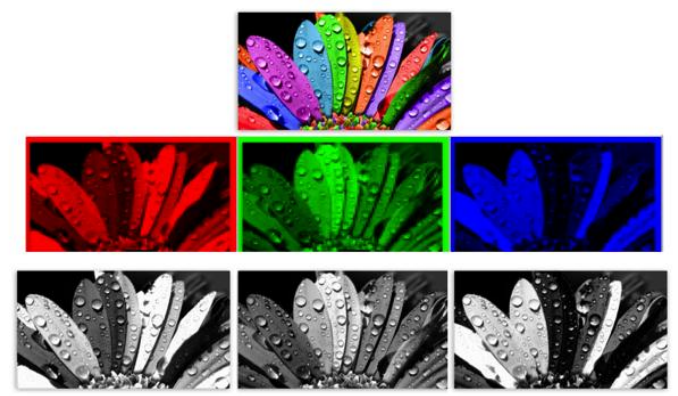

Fig. 4 Independent channels with its grey-scale equivalences.

The basic form of grey-scale image is the RGB scale is calibrated so that the red, green, blue values are equal that has no direction to any hue, means its only contains intensity.

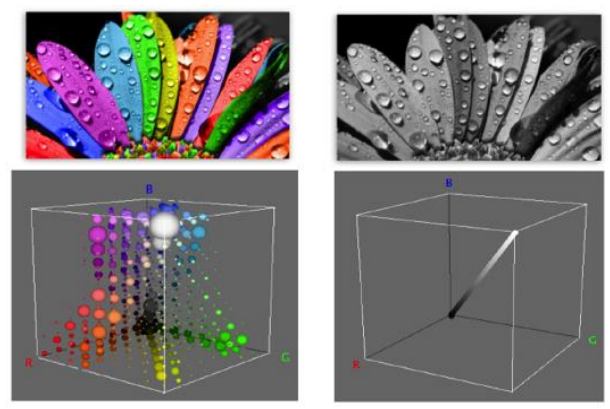

Fig. 5 Red, green and blue value in 3D histogram.

The grey picture is needed in this research in order to get into pseudo-color technique when we start from digital image.

\section{A. Grey Scale Conversion Methods}

Despite many request for grey-scale conversion, in this section we will give a brief explanation about four gray scale conversion methods and later show the implementation result.

Basically grey-scale algorithms utilize three main steps:

1) Read and get the $r, g$ and $b$ values from pixels contains in the image.

2) Use method formula to turn the $r, g$ and $b$ values into single intensity value. 
3) Read back the pixels in order to put the intensity value. When the $\mathrm{r}, \mathrm{g}$, b resulting same values for each pixel, then the conversion succeed.

The basic problem of grey-scale transformation is to recreate the original color include contrast and features [6].

1) Average $=(\mathrm{R}+\mathrm{G}+\mathrm{B}) / 3$.

Average is the most common color to grey-scale method in image processing because it simply averaging the $\mathrm{r}, \mathrm{g}$ and $\mathrm{b}$ values on each pixel [2].

2) Lightness $=(\max (\mathrm{R}, \mathrm{G}, \mathrm{B})+\min (\mathrm{R}, \mathrm{G}, \mathrm{B})) / 2$.

This method averages the highest (maximum) and lowest (minimum) of $r, g$, and $b$ values in the pixels. For example we are searching for maximum values of the whole pixels and pixel (1) is r, g, b $(0,0,150)$ while pixel (2) is r, g, b $(0,0,10)$ then the calculation will set pixel (1) to 150 and pixel (2) to 10 . This is because this method only cares about which color is highest or lowest without considering which channel it comes from [2].

3) Lumino/Luminance $=0.2126 \mathrm{R}+0.7152 \mathrm{G}+0.0722 \mathrm{~B}$.

The luminosity method averages the hman perception value. Humans perceive green more strongly than red, and red more strongly than blue, therefore green is weighted heavily compare to red and blue.

4) RGB color space converted to YPQ color space.

YPQ color space was introduced in [7] to convert color to gray scale image by fusing the information from the luminance and chrominance channels. RGB color space can be converted into YPQ color space using (1):

$$
\left[\begin{array}{l}
Y \\
P \\
Q
\end{array}\right]=\left[\begin{array}{ccc}
0.2989 & 0.5870 & 0.1140 \\
0.5000 & 0.5000 & -1.0000 \\
1.0000 & -1.0000 & 0.0000
\end{array}\right]\left[\begin{array}{l}
R \\
G \\
B
\end{array}\right]
$$

With Y represent luminance channel, P for yellow-blue and $\mathrm{Q}$ for red-green [5].
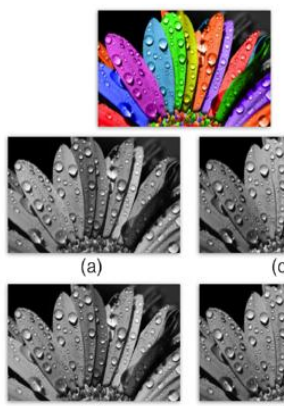

(b)

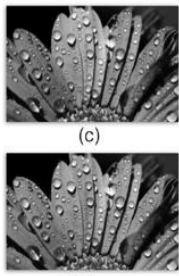

(d)

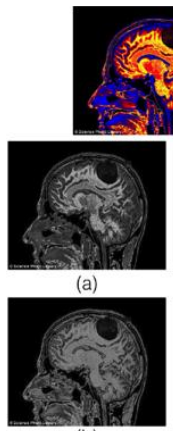

(b)

palette card contained 2 colors and the third palette card contained only 1 color.

2) Each of these palette card have corresponding 4 cards with the result according to the four color to grey conversion methods described above.

3) The subject then will be asked to choose the palette card from one color, two colors then last is five colors.

4) Subjects will rank the cards based on which grey cards that presents their opinion of colors in palette card from most fitting to least fitting. As seen in Fig. (3), the grey cards have code number on the left up; each number represent 1:Average, 2:Lightness, 3:Lumino and 4:YPQ.
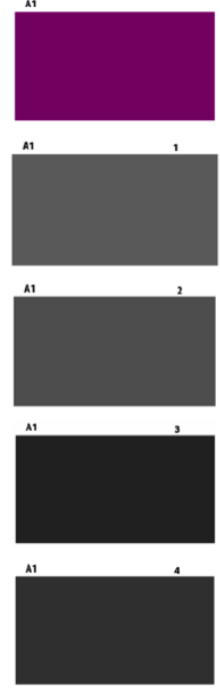
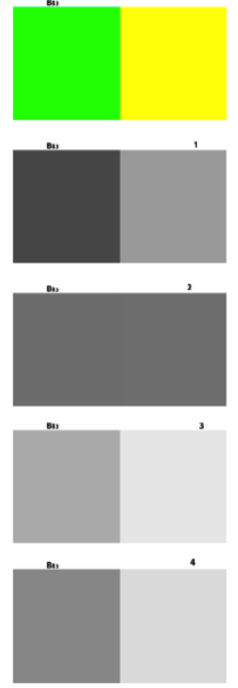
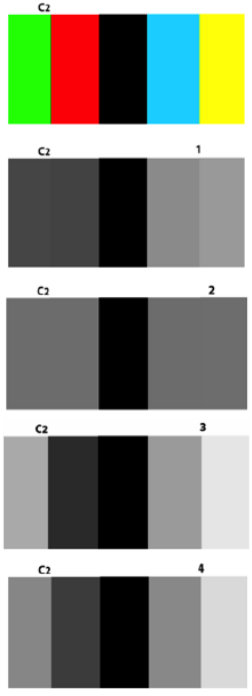

Fig. 7 Palette cards with its conversion cards.

Clearly there are lots of issues to consider on calculating the result of the questionnaire. There may not be one method can decide to be the best, but we can make an approach using voting method that mostly used to calculate candidate's debate and election from social-political area [8]. Below is the explanation of three well known voting methods.

1) Winner Method

This is the easiest voting method where we calculate only on how many people chooses the particular method.

2) Borda Count

Borda count is also called single-winner voting method where the subject will rank the options by numbering the options to the position in the ranking based on rule below: If $N$ is the number of candidates then,

Each 1st-place is worth $N$ points.

Each 2nd-place is worth $N-1$ points.

Each third-place rank is worth $N-2$ points.

Each $N_{\text {th }}$-place (last-place) rank is worth 1 point.

Because we have four color to grey conversion methods then this will lead us to determine 4 point for winner, 3 for the second, 2 for the third and 1 for least preferred method.

3) Copeland Method

Copeland method is one of the Condorcet method in which the winner is determined by finding the candidates with the most pairwise winning [10].

1) For the initial experiment, we prepared 3 palette cards; the first palette card contained 5 different colors, the second 
The calculation then started from 1 color palette card until the values for 2 colors palette card and 5 colors palette card. Table 1 will show the result of whole comparison of those three voting methods. As results, 39 users elected YPQ method as the best conversion technique, which means this color space would be the best choice to be used for Pseudo-color technique.

TABLE I

VOTING RESULT FOR COLOR TO GREY CONVERSION METHODS

\begin{tabular}{cc}
\hline \hline & One Color \\
\hline Winner Method & $4>3>2>1(14: 12: 7: 6)$ \\
Borda Count Method & $4>3>1>2(108: 102: 93:$ \\
& $91)$ \\
Copeland Method & $4>3>2>1>2(7: 5: 2: 4)$ \\
\hline & Two Colors \\
\hline Winner Method & $3>1>2>4(14: 11: 10: 4)$ \\
Borda Count Method $\quad 4>1>3>2(106: 101: 100:$ \\
$\quad 89)$ \\
Copeland Method $\quad 4>3=1>2(6: 5: 5: 2)$ \\
\hline \multicolumn{2}{c}{ Five Colors } \\
\hline Winner Method & $4=2=1>3(10: 10: 10: 9)$ \\
Borda Count Method & $4>1>3>2(109: 108: 94:$ \\
& $80)$ \\
Copeland Method & $1>4>3>2(7: 6: 5: 0)$ \\
\hline \hline
\end{tabular}

\section{PSEUDO-COLOR TECHNIQUE}

Pseudo-color processing is a technique that maps each of the grey levels of a black and white image into an assigned color. This colored image, when displayed, can make the identification of certain features easier for the observer. The mappings are computationally simple and fast. This makes pseudo-color an attractive technique for use on digital image processing systems that are designed for interactive mode [9].

The intensity value on grey image can be described with LUT that gives the value from 0 to 255 for red, green and blue. To find out the fracture and main object in an image, the particular amount of colors will replace the grey/intensity values. At the end, each color in pseudo-color stands for intensity differences that grey image has.

Overall, the system of pseudo-color can be described as below

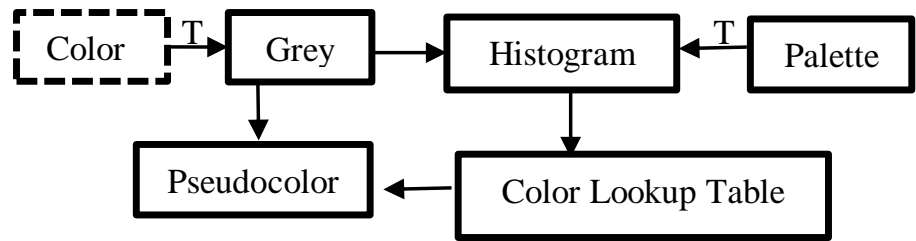

Fig. 8 Pseudo-color process. "T" shows steps where a color to grey conversion is needed.

\section{A. Histogram of Image Intensity}

Pixels on image can be represented as an array. The grey-scale image will have same intensity values from 0 (black) to 255 (white) and the values between are various shades of grey. Basically image contains red, green and blue value. For color image, these values are different but for grey image red, green and blue will be the same.

As in Fig.5 we can see that color image can be well described in $3 \mathrm{D}$ histogram where each vector belongs to one color. In grey image, we are focusing on the histogram that represents the number of pixels on the $y$-axis and the 256 different possible intensities on the $x$-axis Fig.9.
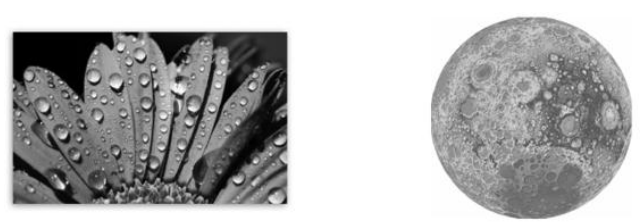

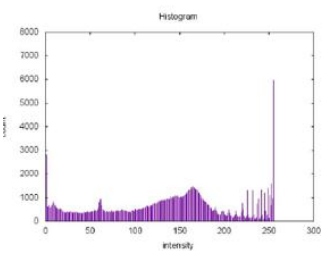

(a)

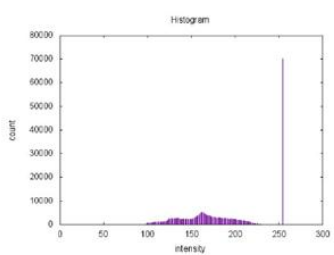

(b)
Fig. 9 Histogram shows the range of intensity.

\section{B. Segmentation and Threshold}

In order to be able to map the color palette into a grey image, we need to classify range of intensity into five highest ranges (because five colors in a palette). Performing clustering-based threshold or Segmentation can do this job. Segmentation is a technique that separates the main object on the image with its background. This helps us to find the region and other properties that won't be necessary. This technique also helps on identify contours by identifying differences between regions (edges) [11].

Threshold is a necessity when we want to segment some regions. As explained before, in a grey image pixels will give the intensity information that can be used to separate dark and light regions. Threshold will separate the image and turn all the pixels into two parts. All pixels below threshold value will become 0 and above threshold will become 1 .

Let's say $g(x, y)$ is a threshold regions of $f(x, y)$ at threshold $\mathrm{T}$,

$$
|x|=\left\{\begin{array}{c}
1 \text { if } f(x, y) \geq T \\
0 \text { if Otherwise }
\end{array}\right.
$$

Here we will be using Otsu's threshold [12], which is the threshold where it minimizes the within-class variance of black and white of the thresholded regions.

The within-class variance is the sum of the variances of each cluster.

$$
\sigma_{w}^{2}(T)=w_{0}(T) \sigma_{0}^{2}(T)+w_{1}(T) \sigma_{1}^{2}(T)
$$

Where the class probability $\mathrm{w}_{0,1}$ is computed from $\mathrm{L}$ histogram with [0, L-1] is the range of intensity level. $\sigma_{0}^{2}(T)$ is the variance of the pixels in the background (below threshold) and $\sigma_{1}^{2}(T)$ is the variance of the pixels in the foreground (above threshold), the full explanation can be seen in [12]. 

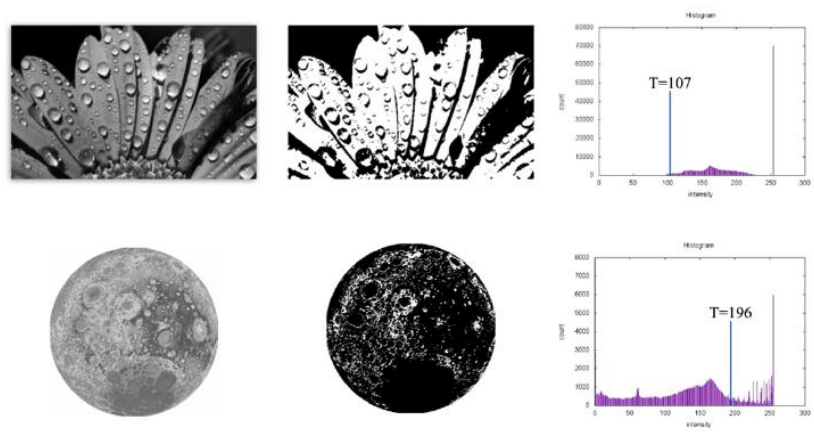

Fig. 10 Otsu's threshold with its threshold value.

In pseudo-coloring, we need to assign arbitrary colors to the grey levels of a grey image. If too many colors are present in the image, it might confuse the observer. Here we are going to use a palette of five base colors.

The RGB value of each color will be used to a create Color Lookup Table. This Color Lookup Table transforms a range of input colors (5 colors) into another range of RGB colors (256 different combinations) by doing color interpolation.

Interpolation is a technique to fill a gap between two numbers [13]. In this topic we are talking about gap between two colors. A smooth transition between two colors can be created by linearly interpolating the color coordinates. Interpolating between colors $a$ and $b$ can be written as

$$
C(t)=C_{a}+\left(C_{b}-C_{a}\right) t
$$

Where $C$ is color interpolation, $a$ is the starting point/first color, $b$ is the ending point/last color and $t$ is between $[0,1]$.

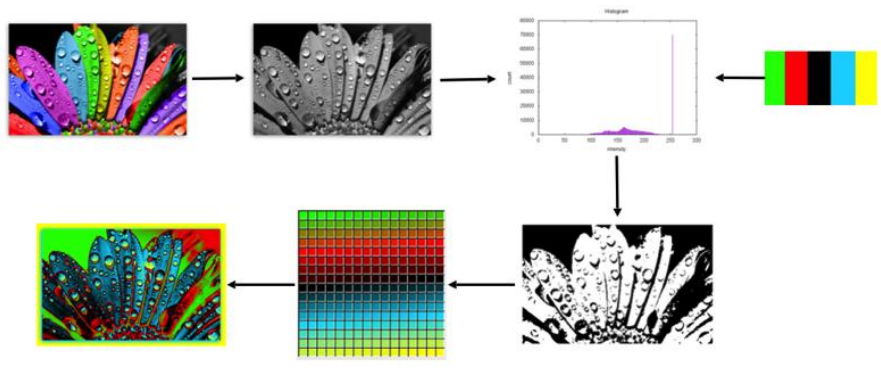

Fig. 11 Pseudo-color technique

\section{V.CONCLUSION}

In this research, we are focusing on Pseudo-color coding that specializes in grey images. The color to grey conversion is needed to be able to do pseudo-color. We presented the experimental study of comparing the suitable color-to-grey conversion method. It should be noted that the result of this study was based on subjective impressions and was calculated using voting calculation method. The result of this subjective impression calculation can be used for further work on Pseudo-color.

Another aim of this research is to show the process on making color reveal the real information of an image, pseudo-coloring will provide additional enhancements and produce an effective visualization for the human.

\section{REFERENCES}

[1] R.N. Czerwinski, D.L. Jones, and W.D. O'Brien, Jr.. "Detection of lines and boundaries in speckle images - Application to medical ultrasound," IEEE Transaction on Medical Imaging 18, pp. 126-136, 1999.

[2] A. Koschan and M. Abidi. Digital Color Image Processing. John Wiley \& Sons, 2008.

[3] Kuo, Sen M., B.H. Lee, and W. Tian. Real-time digital signal processing: fundamentals, implementations and applications. John Wiley \& Sons, 2013, ch. 11.

[4] G. Ira. Processing: creative coding and computational art. Apress, 2007, ch. 4.

[5] J.C. Russ. "The image processing handbook, sixth edition." CRC Press, 2016, ch. 10.

[6] S. Kaleigh, P.E. Landes, J. Thollot, and K. Myszkowski. "Apparent greyscale: A simple and fast conversion to perceptually accurate images and video." Computer Graphics Forum, vol. 27, no. 2, pp. 193-200. Blackwell Publishing Ltd, 2008.

[7] M. Grundland and N.A. Dodgson. Decolorize: Fast, Contrast Enhancing, Color to Grayscale Conversion. Pattern Recognition, vol. 40, no. 11, pp. 2891-2896, Elsevier, 2007.

[8] C. Boergers. Mathematics of social choice: voting, compensation, and division. Society for Industrial and Applied Mathematics, 2009.

[9] Radewan, H. Clark. "Digital image processing with pseudo-color." Acquisition \& Analysis of Pictorial Data. International Society for Optics and Photonics, 1975.

[10] Pomerol, J. Charles, and S.B. Romero. Multicriterion decision in management: principles and practice. Vol. 25. Springer Science \& Business Media, 2012.

[11] B.S. Morse. "Thresholding." Brigham Young University, 1998;2000.

[12] N. Otsu. "A threshold selection method from grey-level histograms," IEEE Transactions on Systems, man, and Cybernetics. Vol. 9, pp. 62-66, 1979

[13] H.C. Lee. Introduction to color imaging science. Cambridge University Press, 2005.

Dwilya Makiwan received her Bachelor of Science (B.sc) from Hasanuddin University, Indonesia in 2013. Now she is a master course student at Department of Creative Informatics, Graduate School of Computer Science and System Engineering, Kyushu Institute of Technology, Japan. Her research interest includes Image Processing focusing on Pseudo-color, Thermography and Bio-inspired Optimization and Learning Algorithms. 